\title{
Path Line Attributes - an Information Visualization Approach to Analyzing the Dynamic Behavior of 3D Time-Dependent Flow Fields
}

\author{
Kuangyu Shi ${ }^{1}$, Holger Theisel ${ }^{2}$, Helwig Hauser ${ }^{3}$, Tino Weinkauf ${ }^{4}$, Kresimir \\ Matkovic $^{3}$, Hans-Christian Hege ${ }^{4}$, and Hans-Peter Seidel ${ }^{1}$ \\ 1 MPI Informatik, 66123 Saarbrücken, Germany \\ \{skyshi,hpseidel\}@mpi-inf.mpg.de \\ 2 Bielefeld University, 33501 Bielefeld, Germany \\ theisel@techfak.uni-bielefeld.de \\ 3 VRVis Vienna, 1220 Vienna, Austria \{hauser, matkovic\}@vrvis .at \\ 4 Zuse Institute Berlin, 14159 Berlin, Germany \{weinkauf, hege\}@zib.de
}

We describe an approach to visually analyzing the dynamic behavior of 3D time-dependent flow fields by considering the behavior of the path lines. At selected positions in the $4 \mathrm{D}$ space-time domain, we compute a number of local and global properties of path lines describing relevant features of them. The resulting multivariate data set is analyzed by applying state-of-the-art information visualization approaches in the sense of a set of linked views (scatter plots, parallel coordinates, etc.) with interactive brushing and focus+context visualization. The selected path lines with certain properties are integrated and visualized as colored 3D curves. This approach allows an interactive exploration of intricate $4 \mathrm{D}$ flow structures. We apply our method to a number of flow data sets and describe how path line attributes are used for describing characteristic features of these flows.

\section{Introduction}

An effective visual analysis of the dynamic behavior of 3D time-dependent flow fields is still a challenging problem in scientific visualization. Although a number of promising approaches have been introduced in recent years, the size and complexity of the data sets as well as the dimensionality of the underlying space-time domain makes the data handling, the analysis and the visual representation challenging and partially unsolved. In particular, it also proves to be inherently difficult to actually comprehend (in detail) the important characteristics of 3D time-dependent flow data. 
In addition to others (streak lines, time lines, etc.), there exist two important kinds of characteristic curves for time-dependent flow fields: stream lines and path lines. While stream lines describe the steady behavior of the flow at a certain time step, path lines describe the paths of massless particles over time in the flow. Hence, the analysis of the dynamic behavior of flow fields is strongly related to the analysis of the behavior of the path lines.

One common approach to analyzing flow fields is to partition the flow domain into areas of characteristically different flow properties. To do so, a variety of different features have been proposed, such as topological features, vortical structures, or shock waves. They reflect different properties of the flow and therefore focus on the representation of different inherent structures. In fact, not all features may give useful information for every flow data set, and the selection of the relevant features is often left to the user in an unsupported way. Moreover, among the features there may be correlations which are either general due to their definition, or they occur in certain areas of particular flows and give relevant information about the behavior of the flow. Therefore we believe that not only the introduction and visualization of new features leads to a deeper understanding of the dynamic behavior of the flow field, but also an effective analysis of the relations between the features and the applications of these results for a visual representation. Our paper is one step along the recently challenging path towards a better understanding of 3D time dependent flow fields.

Our approach starts with the extraction of a number of properties (features, scalar values, and time series) at each point of a regular sampling of the $4 \mathrm{D}$ space-time domain. We have focused on properties describing the (local or global) behavior of the path lines, being either classical and well-established values in vector algebra, or properties newly proposed in this paper. The result of this step is a path line attribute data set: a four-dimensional multivariate data set collecting all computed path line properties.

The visual analysis of multidimensional multivariate data is a well researched topic in information visualization. A variety of techniques has been developed to visualizing such data sets making inherent correlations visible. Because of this we attempt to use information visualization approaches to analyzing the path line attributes data set. The results of this analysis (i.e., selections of path lines with certain combinations of properties) are then used for a focus+context visualization of either the selected path lines or the interesting properties. This way the user is able to do a simultaneous exploration in the $4 \mathrm{D}$ space-time domain of the flow and in the abstract path line attribute space. We show that this can give new insight into characteristic substructures of the flow which leads to a better understanding of time-dependent flow fields.

The rest of the paper is organized as follows. Section 2 mentions related work in the visualization of 3D time-dependent flow fields. Section 3 presents the properties of path lines which we extract for the further analysis. Section 4 describes our information visualization approach and explains how to use 
it for a focus+context visualization of the flow data. Section 5 applies our approach to a number of data sets. Section 6 draws conclusions and mentions issues of future research.

\section{Related Work}

The idea to segment a flow domain into areas of certain flow properties has been used for 3D steady flow fields for a variety of features, such as topological features [GLL91, LDG98, MBSHJ04, TWHS03, WTHS04] or vortex regions [Hun87, SH95, PR99]. [SS05] provides a general framework of this in the context of topological features. [PVHLD03] gives an overview on flow visualization techniques focusing on feature extraction approaches.

The extension of these techniques to 3D time-dependent fields is usually done by observing the feature regions over time, see [TSH01, TS03, GTS04] for topological features and [BS94, BS95, BP02, TSWHS05] for vortex features. Although these approaches provide insight into the flow behavior at arbitrary time steps, the analysis of the dynamic behavior based on path lines make specialized approaches necessary. [WS05] visualizes a number of carefully selected path lines to get static representations of the dynamic flow. [TWHS05] considers a segmentation of the flow domain based on local properties of the path lines. [WEE03, WSEE05] apply texture based visualization approaches to capture path line characteristics.

The idea of connecting information visualization and scientific visualization approaches is considered to be one of the "hot topics" in visualization [Joh04]. Salzbrunn et al. published an approach of streamline predicates for steady flow [SS05]. The work closest to ours is the SimVis approach [DGH03, DMGPH05] which uses approaches of information visualization to analyzing various kinds of simulation data. The main difference to our approach is that SimVis works on multiple scalar data describing certain properties of the simulation. Contrary to this, our approach works on dynamic flow data, focusing on local and global properties of path lines, i.e. on a multi-variate properties data set, derived from a 3D unsteady flow field.

\section{Path Line Attributes}

Given a 3D time-dependent vector field $\mathbf{v}(\mathbf{x}, t), \mathbf{x}$ describes the 3D domain and $t$ is the temporal component. Stream lines and path lines are generally different classes of curves [TWHS04]. Stream lines are the tangent curves of $\mathbf{v}$ for a fixed time $t$, while path lines describe the paths of massless particles in $\mathbf{v}$ over time.

Given a point $(\mathbf{x}, t)$ in the space-time domain, the stream line starting at $(\mathbf{x}, t)$ can be written in a parametric form 


$$
\mathbf{s}_{\mathbf{x}, t}(\tau)=\mathbf{x}+\int_{0}^{\tau} \mathbf{v}\left(\mathbf{s}_{\mathbf{x}, t}(s), t\right) d s
$$

while the path line starting at $(\mathbf{x}, t)$ has the parametric form

$$
\mathbf{p}_{\mathbf{x}, t}(\tau)=\mathbf{x}+\int_{0}^{\tau} \mathbf{v}\left(\mathbf{p}_{\mathbf{x}, t}(s), s+t\right) d s .
$$

Path lines depict the trajectory of massless particles in a time-dependent flow. To characterize path lines, we consider two kinds of information: scalar values that describes local or global properties of a path line, and time series that collects information along a path line.

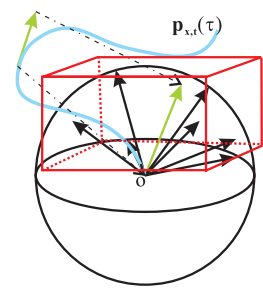

(a)
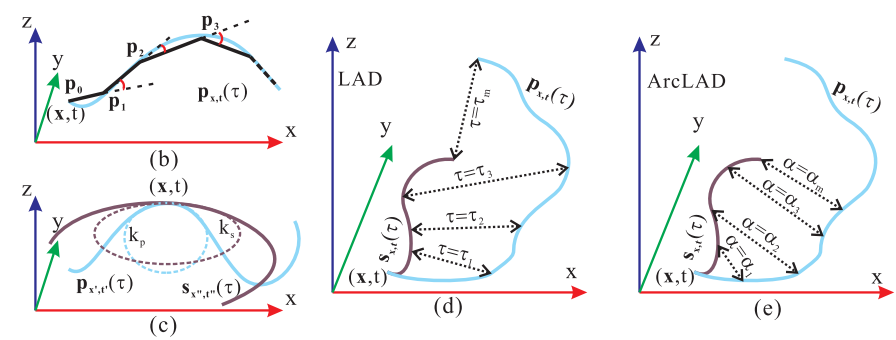

Fig. 1. a) Mapping the direction vectors along a path line to a unit sphere and calculating the bounding box approximation of the opening cone; b) Winding angle along a path line; c) Curvature difference between the path line and stream line pass through a specified point. d) LAD that records the Euclidean distance between the point of a path line the corresponding stream line at the same time $\tau$; e) ArcLAD that records the Euclidean distance between the point of a path line and the corresponding stream line at the same arc length $\alpha$ from the start point.

For scalar attributes, we compute a number of scalar properties of the path line starting at a given point $(\mathbf{x}, t)$ which reflect either local or global properties of the path lines. In the latter case, the value depends on the considered integration time. Since we are interested in the global behavior of the path lines, the integration time can be chosen rather large (relative to the time interval in which $\mathbf{v}$ is defined). In particular, we compute the scalar values in Table 1.

For time series we have investigated the attributes in Table 2.

\section{System overview}

Fig. 2 shows the pipeline of our path line attribute analysis approach. We start with a 3D time-dependent flow field $\mathbf{v}$ to be analyzed. As a first step, we apply a sampling of the space-time domain to obtain the points for which we compute the path line attributes. Note that since the data lives in a $4 \mathrm{D}$ 
Table 1. Scalar Attributes

\begin{tabular}{|c|c|c|}
\hline Id & Name & Description \\
\hline & Non Straioht Velocity & $\int_{0}^{\tau}\left\|\mathbf{v}\left(\mathbf{p}_{\mathbf{x}, t}(s), s+t\right)\right\| d s-\left|\mathbf{p}_{\mathbf{x}, t}(\tau)-\mathbf{x}\right|$ \\
\hline nonstraightV & Non straight Velocity & $\overline{\left|\mathbf{p}_{\mathbf{x}, t}(\tau)-\mathbf{x}\right| \quad \tau}$ \\
\hline distSE & Relative start end distance & $\mathrm{p}_{\mathrm{x}+\mathrm{t}^{\tau}(\tau)-\mathrm{x}}$ \\
\hline avDir & Average direction & $\frac{\mathbf{p}_{\mathbf{x}, t}(\tau)-\mathbf{x}}{\left\|\mathbf{p}_{\mathbf{x}, t}(\tau)-\mathbf{x}\right\|}$ \\
\hline av ParticleV & Average particle velocity & $\frac{\int_{0}^{\tau}\left\|\mathbf{v}\left(\mathbf{p}_{\mathbf{x}, t}(s), s+t\right)\right\| d s}{\tau}$ \\
\hline lyapunov & Lyapunov exponent & $\frac{\log \left(\sqrt{\left.\lambda_{\max }\left(A^{T} A\right)\right)}\right.}{\mathrm{SLM} 05]}, A=\nabla_{\mathbf{x}} \mathbf{p}_{\mathbf{x}, t}(\tau)[\mathrm{GRH} 07$, \\
\hline wind & Winding Angle & $\sum_{i=0}^{n-2} L\left(\left(\mathbf{p}_{i+1}-\mathbf{p}_{i}\right),\left(\mathbf{p}_{i+2}-\mathbf{p}_{i+1}\right)\right)$, Fig. $1 \mathrm{~b}$ \\
\hline lad & Local acceleration displacement & $\left\|\mathbf{p}_{\mathbf{x}, t}(\tau)-\mathbf{s}_{\mathbf{x}, t}(\tau)\right\|$, Fig. 1d \\
\hline curvDiff & Curvature difference & $\left(\kappa_{\mathbf{S}}-\kappa_{\mathbf{p}}\right)^{2}, \kappa_{\mathbf{p}}=\frac{\|\dot{\mathbf{p}} \times \ddot{\mathbf{p}}\|}{\|\dot{\mathbf{p}}\|^{3}}, \kappa_{\mathbf{S}}=\frac{\|\dot{\mathbf{s}} \times \ddot{\mathbf{s}}\|}{\|\dot{\mathbf{s}}\|^{3}}$, Fig $1 \mathrm{c}$ \\
\hline div & Local divergence & $\operatorname{div}(\mathbf{v})$ \\
\hline
\end{tabular}

Table 2. Time Series Attributes

\begin{tabular}{lll}
\hline Id & Name & Description \\
\hline DistEu & Euclidean distance to start & Dist $E u(\tau)=\left\|\mathbf{p}_{\mathbf{x}, t}(\tau)-\mathbf{x}\right\|$ \\
$L A D$ & Local acceleration displacement & $L A D(\tau)=\left\|\mathbf{p}_{\mathbf{x}, t}(\tau)-\mathbf{s}_{\mathbf{x}, t}(\tau)\right\|$, Fig. 1d \\
$A r c L A D$ & Arc local acceleration displace- & Fig. 1e \\
& ment & \\
Dir & Direction vector & Fig. 1a \\
OpeningCone & Opening cone & \\
Curvature & Curvature & \\
Velocity & Velocity & \\
\hline
\end{tabular}

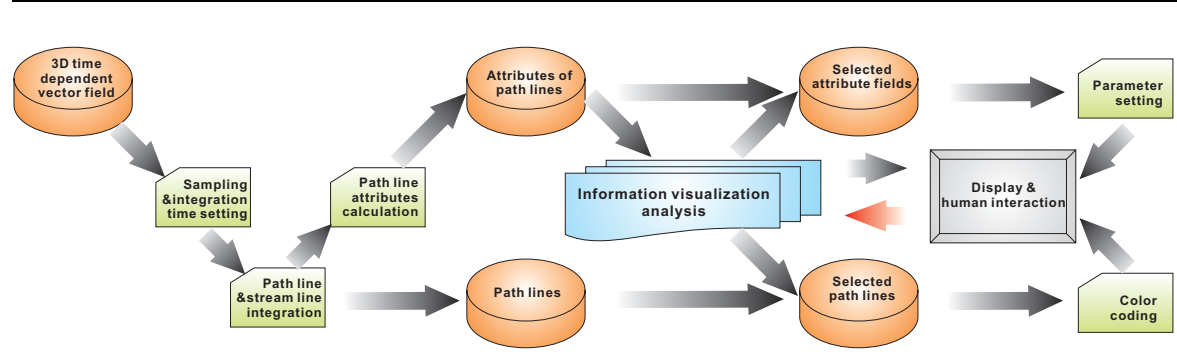

Fig. 2. Pipeline for analyzing path line attributes.

domain, even a rather small sampling density may give a high amount of sample points. Therefore, the sampling density should be a compromise between the spatio-temporal accuracy of the analysis and the available computing resources. If the analysis delivers interesting features in certain smaller regions of the domain, this region can be analyzed using a higher sampling density to make sure the sampling rate is above the Nyquist frequency . At this state of the approach we also have to set the integration time for the path lines. Also this setting is a tradeoff between the fact that we want to have the path lines to be analyzed as long as possible and the property that most of the path lines should be integrated over the same time without leaving the domain. 
The next step of the approach is the integration of the stream lines and path lines starting from the sampled points over the set integration time. For our examples we have used a 4th order Runge-Kutta integration. From these integrations we compute all path line attributes introduced in section 3 .

The set of all path line attributes is the input of our information visualization core module which will be described in section 4.1 in more detail. Interactive visual analysis on the basis of state-of-the-art information visualization techniques and brushing in linked views is used to extract relevant correlations, interesting feature combinations, or general properties of the data. Note that the brushed features are not necessary physical variable. The result of this analysis is used to steering the visualization of the path lines and their attributes. If the interactive visual analysis delivers interesting features in a certain scalar path line attribute, we can visualize it using standard volume rendering techniques like direct volume rendering or slicing. Furthermore, the interactive visual analysis delivers a selection of interesting path lines having a certain combination of properties. They are visualized as 3D line structures with a color coded time component.

Our implementations of the visualization of the selected path lines and the selected attributes are based on Amira [SWH05], whereas our information visualization analysis is based on the ComVis system which is described in section 4.1 .

\subsection{The ComVis system}

ComVis is an interactive visualization tool. It supports conventional information visualization views such as $2 \mathrm{D}$ and $3 \mathrm{D}$ scatter plots, parallel coordinates, histograms, as well as a special curves view which is used for displaying function graphs. This combination of views makes it possible to analyze a wide variety of data where in the same row of a multi-variate table some values are scalar (just as it is usual) and others correspond to a function graph (common in various kinds of scientific data)[MJJKG05]. The tool offers multiple linked views parallel to each other. Each view can be of any of the above mentioned view type. ComVis pays great attention to interaction. Due to advanced brushing and linking proved to be very powerful analytical tool. Users can brush the visualized data in any view, all linked views reflect the data selections by appropriate focus+context visualization. Furthermore, the user can use a simple, yet powerful line brush in the curves view. The line brush selects all curves which intersect the line. All brushes can be scaled and moved interactively. The multiple brush mode makes it possible to flexibly combine various brushes. The user selects brushes and boolean operations between them. AND, OR, and SUB are supported. Furthermore, the tool creates a composite brush in an iterative manner. This means that the user selects a current operation (AND, OR, or SUB) and draws a brush. The previous brushing state is combined with the new brush accordingly. The new state is computed, and it is used when the user draws another brush. In this 
way the user immediately gets visual feedback, and can very easily broaden the selection (using OR), or can further restrict the selection (using AND or SUB). Once the user is satisfied with a selection (or in the meantime), a tabular representation of the selected data can be shown and exported to file on demand.

\section{Applications}

We applied our approach to a number of data sets. Not surprisingly, not all attributes are interesting in all data sets, and different path line attributes turn out to be important for different data set. However, we can also identify several interesting coherencies between different path line attributes which seem to hold even for different data sets. Accordingly, we are optimistic that the here described analysis indeed provides a useful basis for future generalization of this approach.

\subsection{D time-dependent cylinder flow}
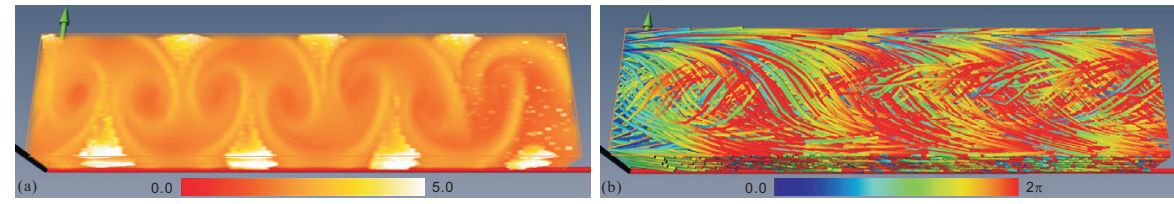

Fig. 3. Flow behind a cylinder: a) Direct volume rendering of the lyapunov attribute field at time 0; b) All considered path lines.

Figures 3 and 4 present some results of analyzing a 3D time-dependent flow behind a circular cylinder. The cylinder is put in the origin with radius 0.5 and height 8.0 , while the data set domain $D$ is $[3.15,19.74] \times[-2.06,2.06] \times$ $[0.09,1.89] \times[0,2 \pi]$. This data set was kindly provided by Gerd Mutschke (FZ Rossendorf) and Bernd R. Noack (TU Berlin). We considered path lines at a $28 \times 14 \times 7 \times 6(191 \mathrm{MB}$ attribute file to ComVis) sampling and used an integration time of $1.5 \pi$ (for the data set given in a $2 \pi$ time slab). Figure $3 \mathrm{a}$ shows the direct volume rendering of one of the attribute fields lyapunov. In figure $3 \mathrm{~b}$, all path lines integrated from the sampled points are displayed. As we can see from figure $3 \mathrm{a}$, there are certain patterns in the lyapunov attribute field. Low lyapunov values indicate stability of the path line. We use the information visualization approach to select the area with low lyapunov, as shown in the upper left of figure 4a. The visualization of the selected path lines is shown in figure 4b. Fig. 4c shows the seed area of the selected path lines at the time 0 . 

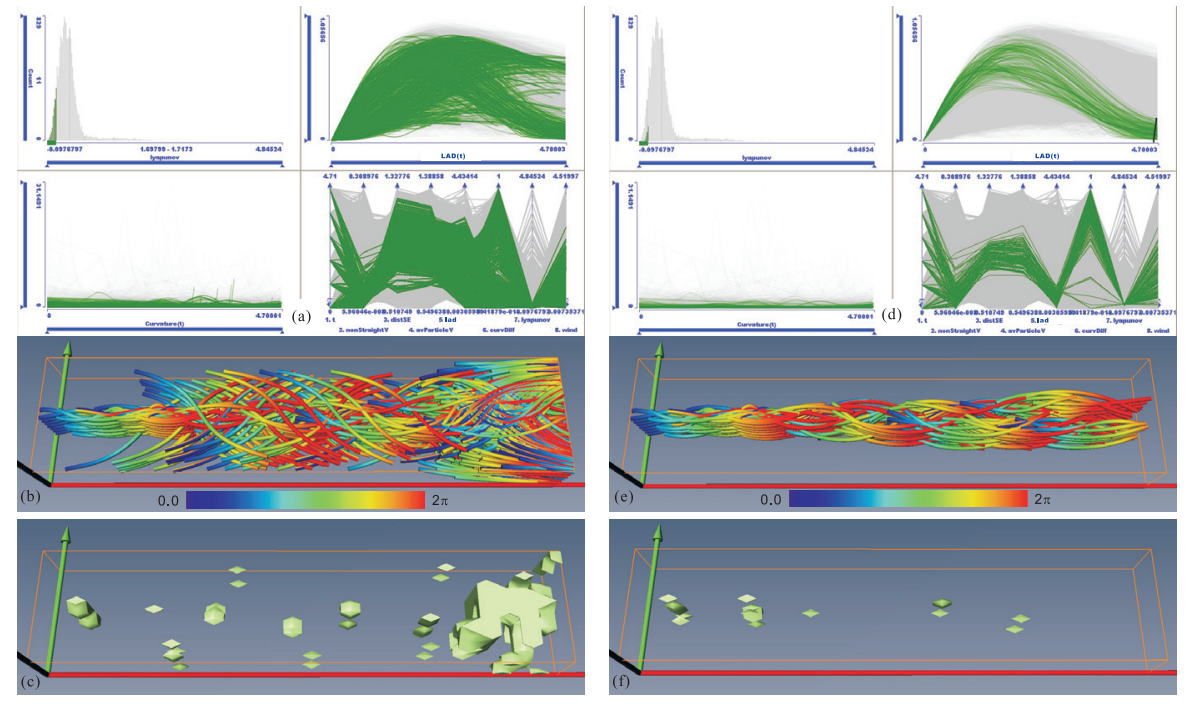

Fig. 4. Feature low lyapunov and $L A D$ : a) Selecting low lyapunov area in ComVis; b) Visualization of selected path lines with low lyapunov; c) Visualization of the seeding area of the selected low lyapunov path lines at time 0; d) Selecting low lyapunov and parabola $L A D$ area in ComVis; e) Visualization of selected path lines with low lyapunov and low $L A D ; \mathrm{f}$ ) Visualization of the seeding area of the selected low lyapunov and low $L A D$ path lines at time 0 .

When investigating the visualized result, we can see that there are further different patterns in the low lyapunov path lines. It is obvious when we investigate the ComVis result of time series $L A D$, after choosing the cluster as shown in the upper right of figure $4 \mathrm{~d}$. We get the path line cluster whose $L A D$ time series have small values at the end of the integration time. Fig. $4 \mathrm{e}$ and $4 \mathrm{f}$ present the visualization of the selected path lines and their seed areas. We notice that they stay in the middle of the domain and along the flow direction directly behind the cylinder.

\subsection{Hurricane Isabel}

Fig. 5 shows a visual analysis of the hurricane Isabel data set, which has been previously analyzed in a number of papers [GM04, DMH04]. We sample the domain with path lines at a resolution of $24 \times 24 \times 6 \times 6(253 \mathrm{MB}$ attribute file to ComVis), and set the maximum integration time to 30 hours (the whole data set covers 48 hours). Fig. 5 a shows the visualization of all considered path lines. Fig. 5b show a direct volume rendering of nonStraight $V$ at time 0 (the starting time of the simulation).

For this data set, we start the information visualization analysis, with the observation of the avParticleV vs. distSE scatter plot (upper right of Fig. 5c), 
showing a number of points on the diagonal but also a number scatter points clearly above it. We expect the points on the diagonal to represent path lines with a rather straight-line-like behavior, whereas the locations of the points above the diagonal may indicate a swirling behavior. Since nonStraight $V$ is equivalent avParticle $V$ vs. distSE, we selected all points above the diagonal, by considering points with a rather high nonStraightV (upper left of Fig. 5c). The parallel coordinate representation (lower right of Fig. 5c) shows that the selected path lines have a rather low curvDiff. This indicates that in these regions stream lines and path lines are locally rather similar. The curvature plot of the selected path lines doesn't have extreme values (lower left of Fig. 5c). The selected path lines are visualized in Fig. 5d, clearly showing that we have selected the ones swirling around the moving eye of the hurricane. Fig. 5 e shows the areas where the selected path lines originate at time $t=0$, while Fig. 5f shows the starting areas of the selected path lines for all time steps.

\subsection{Airfoil}

Figures 6 - 7 show a comparative visual analysis of 8 different data sets of a flow around an airfoil. The difference between these 8 data sets are the air injection frequency. The injection frequencies are 0 (base), 0.2, 0.44, 0.6, $0.88,1.0,1.5$ and 2.0. The goal of our analysis is to find the best air injection frequency which contributes the best lift power. It is known that abnormal vortex structures reduce the lift of the airfoil. Therefore, our visual analysis focuses on the areas with vortices where the probability of abnormal flow is high. We reduce our consideration to a small area around the areas with vortices.

We sample the interesting area with path lines at a resolution of $36 \times 12 \times$ $8 \times 10$ for each data set, and set the maximum integration time to 30 seconds (the whole data set covers different time domains for different frequencies and the path line integration will usually leave the domain within 30 seconds for each frequency). Fig. 6 shows the visualization of all considered path lines for different frequencies. We observe that most path lines behave well showing a rather straight behavior. The abnormal flows correspond to those non straight path lines. As our experience on these attributes, the nonStraightV is a good attribute to reflect the characteristics of straightness of path lines. So we compare this attribute computed at same location and same time for different frequency data sets in ComVis.

Fig. 7 shows the comparative result of the analysis of the nonStraightV for these 8 different frequencies. Relative analysis is popular in airfoil analysis since the relative flow behavior for different parts of an airfoil determines the lift power. We apply a relative selection here and select those path lines for each data set with 70 percent highest nonStraightV attributes. Those selected path lines and the corresponding seeding areas are visualized. We can see that these selected non straight path lines are closed to the area with vortices. And we can clearly observe that for a frequency 0.6 , there are fewest non straight 
path lines and the non straight seeding areas are the smallest. So we find that for frequency 0.6 , the probability of abnormal flow is less compared to others. We have tested several other percentage of the highest nonStraightV. All the results present the equivalent information. We conclude that 0.6 is the best air injection frequency among the 8 tests. The experience from the industry partner confirms this result successfully.

\section{Conclusions}

To getting insight into the dynamic behavior of path lines of 3D timedependent flow fields is still a challenging problem for the visualization community. Path lines elude a straightforward extension from stream line based methods because path lines can be integrated only over a finite time, and they may intersect each other (at least when only considering their 3D reference locations). This paper is the - to the best of our knowledge - first approach to getting insight into the behavior of path lines by applying an approach from information visualization. In particular, we made the following contributions:

- We identified a number of local and global attributes of path lines which we expect to contain relevant information about the path line behavior.

- We interactively analyzed these attributes by using an approach from information visualization. The results were used to steering a 3D path line visualization.

- We applied our approach to a number of data sets, in order to get new insight into the path line behavior.

During our analysis it turned out that not all path line attributes gave useful results for all data sets. However, inherent and data independent correlations in the attribute data set can be expected, making a reduction of the attribute set possible. In particular, we have the impression that the investigation of path line attributes can indeed lead to a useful and practicable way of accessing/segmenting interesting flow features in time-dependent data sets, including swirling/vortical/rotating flow subsets, (e.g., via attributes wind and nonStraight $V$ ), quasi-steady flow structures, (e.g., via attributes $L A D$ and $\operatorname{ArcL} A D$, etc.), etc. We are optimistic with respect to these expectations, not at the least because it was, for example, fairly straight forward and quite easy to accomplish to extract the rotating main vortex of hurricane Isabel, which - to the best of our knowledge - cannot so easily be accomplished with any of the previously published vortex extraction methods.

\section{Acknowledgments}

We thank Bernd R. Noack for the supply of the cylinder data which was kindly provided by Gerd Mutschke. The Hurricane Isabel data produced by 
the Weather Research and Forecast (WRF) model, courtesy of NCAR and the U.S. National Science Foundation (NSF). Also many thanks to Jan Sahner for support and fruitful discussion. Parts of this work have been done in VRVis Research Center which is funded by an Austrian research program called Kplus. Also, parts of the work was funded by the Max-Planck center of visual computing and communication. All visualization of this paper have been created using ComVis (see http://www.vrvis.at/via/products/comvis.html) and Amira - a system for advanced visual data analysis [SWH05] (see http://amira.zib.de/).

\section{References}

[BP02] Bauer, D., Peikert, R.: Vortex tracking in scale space. In: Proc. VisSym 02 , 233-240 (2002)

[BS94] Banks, D.C., Singer, B.A.: Vortex tubes in turbolent flows: Identification, representation, reconstruction. In: Proc. IEEE Visualization 1994, 132-139 (1994)

[BS95] Banks, D.C., Singer, B.A.: A predictor-corrector technique for visualizing unsteady flow. IEEE Transactions on Visualization and Computer Graphics., 1(2), 151-163 (1995)

[DGH03] Doleisch, H., Gasser, M., Hauser, H.: Interactive feature specification for focus + context visualization of complex simulation data. In: Proc. VisSym 03, 239-248 (2003)

[DMH04] Doleisch, H., Muigg, P., Hauser, H.: Interactive visual analysis of hurricane isabel. VRVis Technical Report (2004)

[DMGPH05] Doleisch, H., Mayer, M., Gasser, M., Priesching, P., Hauser, H.: Interactive feature specification for simulation data on time-varying grids. In: SimVis 05, 291-304 (2005)

[GLL91] Globus, A., Levit, C., Lasinski, T.: A tool for visualizing the topology of three-dimensional vector fields. In: Proc. IEEE Visualization 1991, 33-40 (1991)

[GM04] Gruchalla, K., Marbach, J.: Immersive visualization of the hurricane isabel dataset. (2004)

[GRH07] Green, M.A., Rowley, C.W., Haller, G.: Detection of lagrangian coherent structures in 3D turbulence. Journal of Fluid Mechanics., 572, 111-120 (2007)

[GTS04] Garth, C., Tricoche, X., Scheuermann, G.: Tracking of vector field singularities in unstructured 3D time-dependent datasets. In: Proc. IEEE Visualization 2004, 329-336 (2004)

[Hun87] Hunt, J.C.R.: Vorticity and vortex dynamics in complex turbulent flows. Trans. Can. Soc. Mec. Engrs., 11:21 (1987)

[Joh04] Johnson, C.: Top scientific visualization research problems. IEEE Comput. Graph. Appl., 24(4),13-17, (2004)

[LDG98] Löffelmann, H., Doleisch, H., Gröller, E.: Visualizing dynamical systems near critical points. In: Spring Conference on Computer Graphics and its Applications, 175-184 (1998) 
[MBSHJ04] Mahrous, K., Bennett, J., Scheuermann, G., Hamann, B., Joy, K.: Topological segmentation in three-dimensional vector fields. IEEE Transactions on Visualization and Computer Graphics., 10(2),198-205 (2004)

[MJJKG05] Matkovic, K., Jelovic, M., Juric, J., Konyha, Z., Gracanin, D.: Interactive visual analysis end exploration of injection systems simulations. In: IEEE Visualization 2005, 391-398 (2005)

[PR99] Peikert, R., Roth, M.: The parallel vectors operator - a vector field visualization primitive. In: Proc. IEEE Visualization 1999, 263-270 (1999)

[PVHLD03] Post, F.H., Vrolijk, B., Hauser, H., Laramee, R.S., Doleisch, H.: The state of the art in flow visualization: Feature extraction and tracking. Computer Graphics Forum., 22(4),775-792 (2003)

[SH95] Sujudi, D., Haimes, R.: Identification of swirling flow in 3D vector fields. AIAA Paper. 95,1715 (1995)

[SLM05] Shadden, S., Lekien, F., Marsden, J.E.: Definition and properties of lagrangian coherent structures from finite-time lyapunov exponents in twodimensional aperiodic flows. Physica D., 212, 271-304 (2005)

[SS05] Salzbrunn, T., Scheuermann, G.: Streamline predicates as flow topology generalization. In: Topo-In-Vis 2005 (2005)

[SWH05] Stalling, D., Westerhoff, M., Hege, H.C.: Amira: A highly interactive system for visual data analysis. The Visualization Handbook, 749-767 (2005)

[TS03] Theisel, H., Seidel, H.P.: Feature flow fields. In: Proc. VisSym 03, 141-148 (2003)

[TSH01] Tricoche, X., Scheuermann, G., Hagen, H.: Topology-based visualization of time-dependent 2D vector fields. In: Proc. VisSym 01, 117-126 (2001)

[TSWHS05] Theisel, H., Sahner, J., Weinkauf, T., Hege, H.C., Seidel, H.P.: Extraction of parallel vector surfaces in $3 \mathrm{~d}$ time-dependent fields and application to vortex core line tracking. In: Proc. IEEE Visualization 2005, 631-638 (2005)

[TWHS03] Theisel, H., Weinkauf, T., Hege, H.C., Seidel, H.P.: Saddle connectors - an approach to visualizing the topological skeleton of complex $3 \mathrm{D}$ vector fields. In: Proc. IEEE Visualization 2003, 225-232 (2003)

[TWHS04] Theisel, H., Weinkauf, T., Hege, H.C., Seidel, H.P.: Stream line and path line oriented topology for $2 \mathrm{D}$ time-dependent vector fields. In: Proc. IEEE Visualization 2004, 321-328 (2004)

[TWHS05] Theisel, H., Weinkauf, T., Hege, H.C., Seidel, H.P.: Topological methods for $2 \mathrm{D}$ time-dependent vector fields based on stream lines and path lines. IEEE Transactions on Visualization and Computer Graphics., 11(4),383394 (2005)

[WEE03] Weiskopf, D., Erlebacher, G., Ertl, T.: A Texture-Based Framework for Spacetime-Coherent Visualization of Time-Dependent Vector Fields. In: Proc. IEEE Visualization 2003, 107-114 (2003)

[WS05] Wiebel, A., Scheuermann, G.: Eyelet particle tracing - steady visualization of unsteady flow. In: Proc. IEEE Visualization 2005 (2005)

[WSEE05] Weiskopf, D., Schramm, F., Erlebacher, G., Ertl, T.: Particle and Texture Based Spatiotemporal Visualization of Time-Dependent Vector Fields. In: Proc. IEEE Visualization 2005 (2005)

[WTHS04] Weinkauf, T., Theisel, H., Hege, H.C., Seidel, H.P.: Boundary switch connectors for topological visualization of complex 3D vector fields. In: Proc. VisSym 04, 183-192 (2004) 

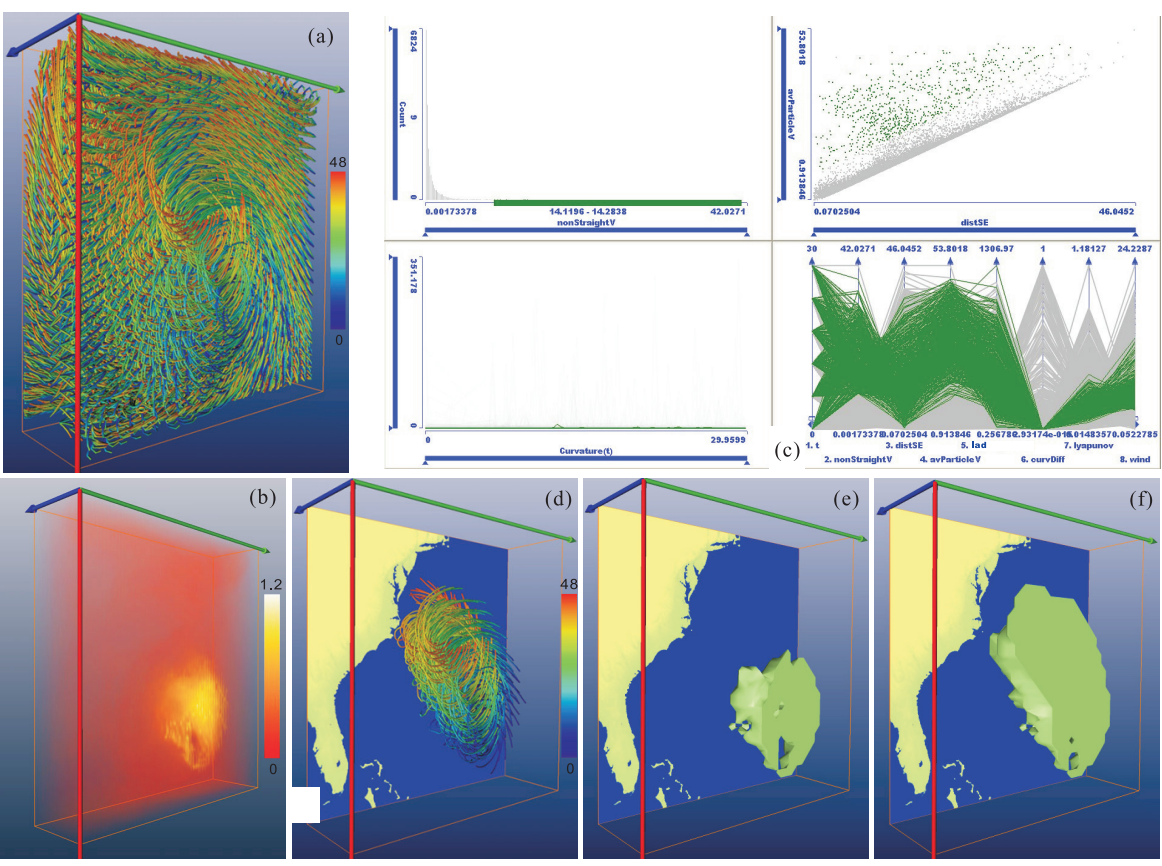

Fig. 5. Analysis and visualization of data set Hurricane Isabel: a)A visualization of all considered path lines. b) Direct volume rendering of the nonStraight $V$ attribute field at time $0 ;$ c) Selecting the area with high nonStraight $V$ which corresponds to swirling behavior in ComVis; d) Visualization of selected path lines of swirling behavior; e) Visualization of the seeding area of the selected swirling path lines at time $0 ; \mathrm{f}$ ) Visualization of the seeding area of the selected swirling path lines at all time steps.
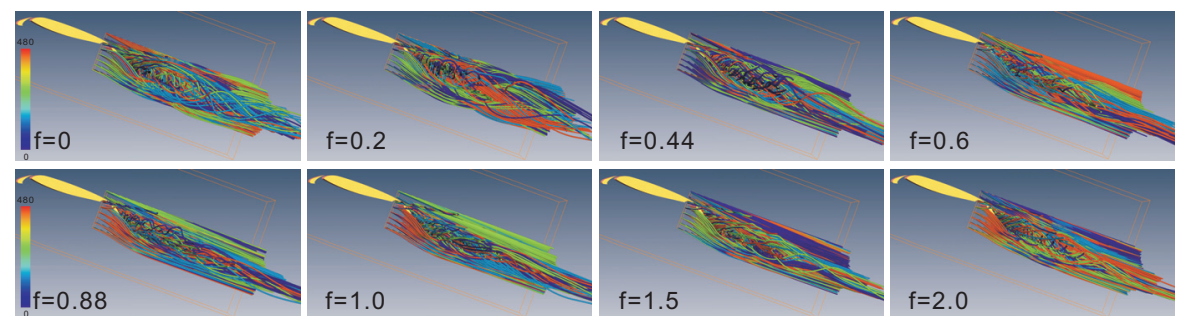

Fig. 6. The path lines started from the focus area of the airfoil flow field for different air injection frequency. 

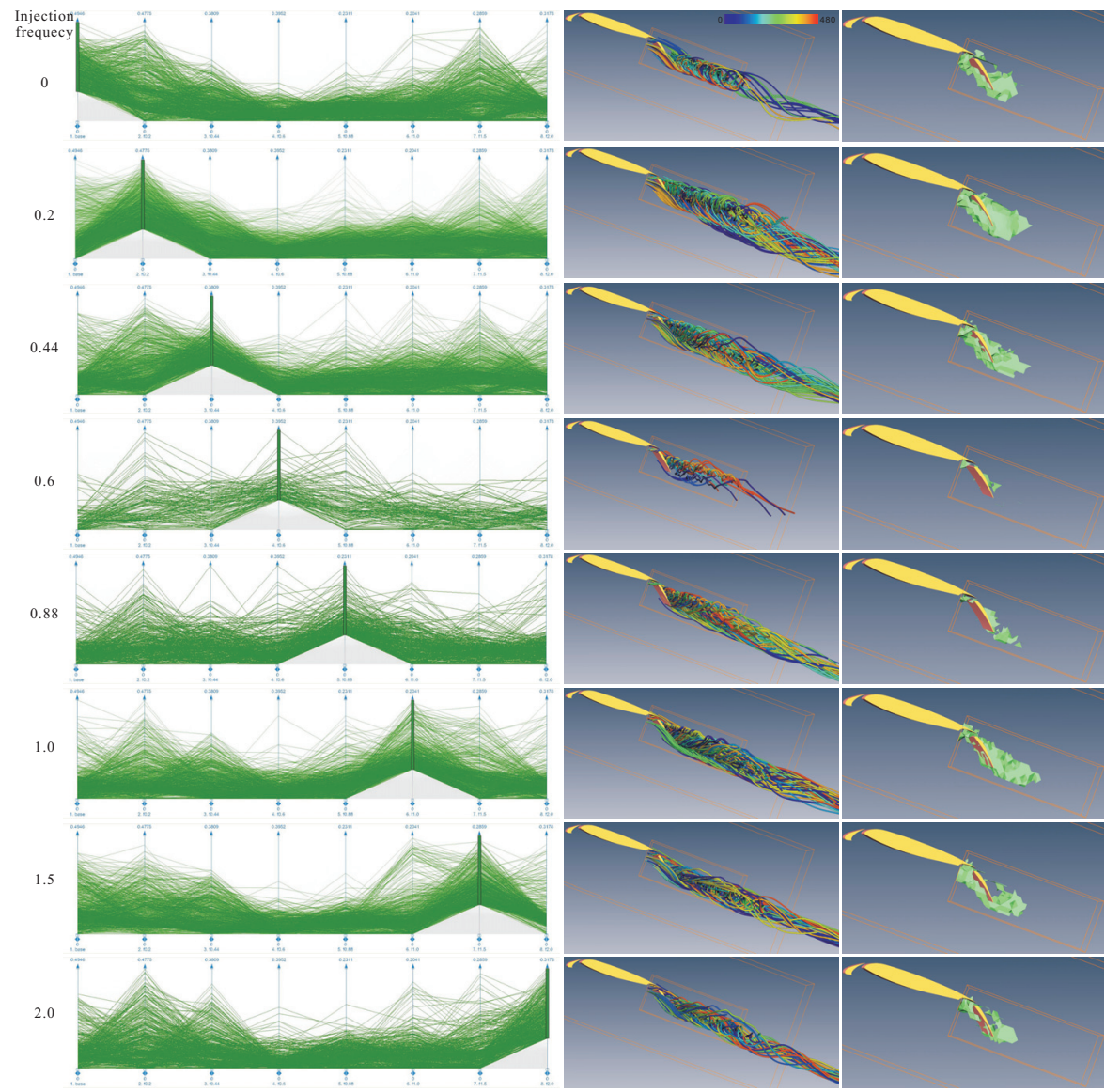

Fig. 7. The comparative analysis result for the attribute nonStraightV of of the airfoil flow field for different air injection frequency. The pictures in the first column depict the selections of 70 percent highest nonStraight $V$ for different frequencies in ComVis. The pictures in the second column depict the corresponding selected path lines for the first column. The pictures in the third column are the corresponding seeding area for the selections in the first column. 